\title{
Special Section: MASCC/ESMO Antiemetic Guidelines
}

Special Guest Editors: Alex Molasiotis, Matti Aapro, Jorn Herrstedt, Richard Gralla \& Fausto Roila

(C) Springer-Verlag Berlin Heidelberg 2016 